\title{
A systematic review of nonsurgical single-visit versus multiple-visit endodontic treatment
}

This article was published in the following Dove Press journal:

Clinical, Cosmetic and Investigational Dentistry

8 May 2014

Number of times this article has been viewed

\section{Amy WY Wong \\ Chengfei Zhang \\ Chun-hung Chu}

Faculty of Dentistry, The University of Hong Kong, Hong Kong Special

Administrative Region, People's

Republic of China
Correspondence: Chengfei Zhang Faculty of Dentistry, the University of Hong Kong, 3B6I, Prince Philip Dental Hospital, 34 Hospital Road, Hong Kong Special Administrative Region, People's Republic of China

Tel +852 2859 037l

Fax +85225599013

Email zhangcf@hku.hk
Abstract: Conventional endodontic treatment used to require multiple visits, but some clinicians have suggested that single-visit treatment is superior. Single-visit endodontic treatment and multiple-visit endodontic treatment both have their advantages and disadvantages. This paper is a literature review of the research on nonsurgical single-visit versus multiple-visit endodontic treatment. The PubMed database was searched using the keywords (endodontic treatment OR endodontic therapy OR root canal treatment OR root canal therapy) AND (single-visit OR onevisit OR 1-visit). Review papers, case reports, data studies, and irrelevant reports were excluded, and 47 papers on clinical trials were reviewed. The studies generally had small sample sizes, and the endodontic procedures varied among the studies. Meta-analysis on the selected studies was performed, and the results showed that the postoperative complications of the single-visit and multiple-visit endodontic treatment were similar. Furthermore, neither single-visit endodontic treatment nor multiple-visit treatment had superior results over the other in terms of healing or success rate. Results of limited studies on disinfection of the root canals using low-energy laser photodynamic therapy is inconclusive, and further studies are necessary to show whether laser should be used in endodontic treatment. This review also found that that neither single-visit endodontic treatment nor multiple-visit treatment could guarantee the absence of postoperative pain. Since the study design of many studies displayed significant limitation and the materials and equipment used in endodontic treatment have dramatically changed in recent years, prospective randomized clinical trials are needed to further verify the postoperative pain and success rates of single-visit versus multiple-visit endodontic treatment.

Keywords: single-visit endodontics, non-surgical endodontics, systematic review, root canal therapy

\section{Introduction}

Endodontic treatment used to take multiple visits to complete, with one of the main reasons for this being that it required a considerable amount of time to complete the treatment. ${ }^{1}$ The use of contemporary endodontics techniques and equipment, such as use of rubber dam, ${ }^{2}$ magnifying devices,${ }^{3,4}$ electronic apex locators, ${ }^{5}$ engine-driven rotary nickel titanium files, ${ }^{6}$ and so forth, not only increases the success rate of endodontic treatment but also, shortens the time needed for the treatment. Endodontic treatment may therefore be completed in a single visit.

The concept of a single-visit root canal treatment was described as early as the $1880 \mathrm{~s}^{7-9}$ Thereafter, there were reports on immediate root filling describing the criteria for success based on the manner of mechanical cleaning and the method of removing the bacterial origins from the canal system. ${ }^{10}$ The treatment techniques used at that 
time were very primitive, and the success rate of single-visit root canal treatment was low. The single-visit treatment was bought back in the 1950 s by Ferranti, ${ }^{11}$ who advocated the use of diathermy for pulpal disinfection and hydrogen peroxide for irrigation. This treatment differed significantly from today's techniques. However, Ferranti was able to describe how the most important criteria for achieving successful results were, in fact, the proper shaping and cleaning of the canals. Currently, these principles are still applied, as important criteria, prior to consideration of single-visit treatment. In 1970, Tosti reported a satisfactory result in his clinical study using a single-visit approach, although the sample size of his study was small. ${ }^{12}$

Nowadays, root canal therapy has become increasingly automated and can be performed more quickly, so some clinicians are incorporating single-visit endodontics into their own clinic routine as a main component of contemporary practice. On the other hand, some dentists believe that the traditional multiple-visit protocol has a long history and a high clinical success rate, preferring to provide multiple-visit endodontic treatment to their patients. This paper aimed to perform a systematic review of clinical studies on the success rate and complications of single-visit endodontic treatment.

\section{Principles of endodontic treatment}

Endodontic treatment, or root canal treatment, entails the removal of the dental pulp and the subsequent shaping, cleaning, and obturation of the root canals of a tooth. The key to endodontic success was described by Gutmann ${ }^{13}$ as the debridement and neutralization of any tissue, bacteria, or inflammatory products within the root canal system. According to the International Conference on Endodontics in 1958, ${ }^{14}$ there are ten important principles to be followed when a dentist performs endodontic treatment on his/her patient. First, endodontic treatment should be conducted using an aseptic technique. Second, the instruments should be confined to the root canal of the treated tooth. Third, the root canal should be prepared using fine and smooth instruments. Fourth, the root canal should be enlarged regardless of its original size, to enable the removal of contaminated dentinal debris and filling of the root canal. Fifth, the root canal should be copiously irrigated with an antiseptic solution during instrumentation. Sixth, the antiseptic irrigation or agents used should be nonirritating to the periapical tissues. Seventh, the sinus tract, if present, should subside after root canal treatment and should not require surgical intervention (however, an incision of the soft tissue can be performed for cases of acute periapical abscess, to allow drainage). Eighth, the canal should be aptly shaped and hermetically obturated. Ninth, a negative culture should be obtained prior to obturation. Finally, the root canal filling should be biocompatible.

Although these principles were applied when performing endodontic treatment, the success rate of endodontic treatment in its early days was not high. With the advance of knowledge and skills and the use of up-to-date material and equipment, endodontic treatment nowadays has become a viable treatment to save the tooth, with a high rate of success. The overall success rate has been reported as being as high as $97 \% .{ }^{15}$ The introduction of the rubber dam was a significant advance in dentistry and in endodontic treatment. ${ }^{16}$ Aseptic technique becomes possible as a result of the introduction of the rubber dam, and therefore, rubber dam isolation is now regarded as a mandatory procedure in the first step of endodontic treatment. Rubber dam isolation prevents ingress of saliva contamination to the root canals. It provides good access for the dentist to work on the tooth. It prevents the accidental swallowing of sharp endodontic instruments. It facilitates thorough cleaning and shaping of the canals. It also increases the patient's and dentist's comfort during endodontic treatment.

The materials and equipment used in endodontic treatment have dramatically changed over the past 20 years. It is not uncommon to relate contemporary endodontics with proper rubber dam isolation, magnifying devices, newer sealants and obturating materials, electronic apex locators, crown-down approaches, ${ }^{17}$ engine-driven rotary nickel titanium files, ultrasonic instrumentation, the copious use of disinfectants and chelating agents, and the latest digital or computer-aided radiographic imaging and three-dimensional (3D) obturation techniques. ${ }^{18}$ Although the materials and equipment differ significantly, the principles remain more or less unchanged. Contemporary endodontic treatment includes the following five principles: 1) use of aseptic technique; 2) cleaning the canals thoroughly and mechanically with the aid of chemical agents; 3 ) shaping the root canals for ease of obturation; 4) obturation to achieve a tight seal of the root canals; and 5) proper restoration of the tooth to prevent coronal leakage, which can induce future bacterial reinfection.

The concept underlying single-visit techniques, as described by Oliet, ${ }^{19}$ is that there is no difference in the treatment criteria to ensure a successful result between multiple-visit and single-visit treatment. The criteria include an accurate diagnosis, proper case selection, and the use of contemporary endodontic techniques. Single-visit endodontic treatment is indicated when both operators and patients want to save chair side time and prefer that anesthetics be adminis- 
tered only once. ${ }^{20}$ Although the treatment can be finished in a single visit, all necessary procedures, such as biomechanical preparation, thorough cleaning, and disinfection, followed by complete obturation of the prepared root canals, should not be compromised. When the tooth is nonvital and there is acute inflammation, single-visit endodontic treatment should not be recommended. ${ }^{20}$ Moreover, patients who have temporomandibular disorders and/or who cannot endure long treatment period may also not be suitable for single-visit endodontic treatment.

\section{Literature search of clinical trials}

A literature review was performed to find descriptions of nonsurgical single-visit endodontic treatment, using the PubMed database, a free search engine primarily accessing the MEDLINE database of references and abstracts on the life sciences and biomedical topics. The keywords used were (endodontic treatment OR endodontic therapy OR root canal treatment OR root canal therapy) AND (single-visit OR one-visit OR 1-visit). The titles and abstracts of articles written in English were screened (Figure 1). A total of 200 publications were identified, and 161 papers were excluded because they were review papers, case reports, data studies, or irrelevant reports. There were 39 papers on clinical trials, and the full texts of these publications were retrieved. A manual search was performed on the references of these papers, leading to the discovery of eight additional publica-

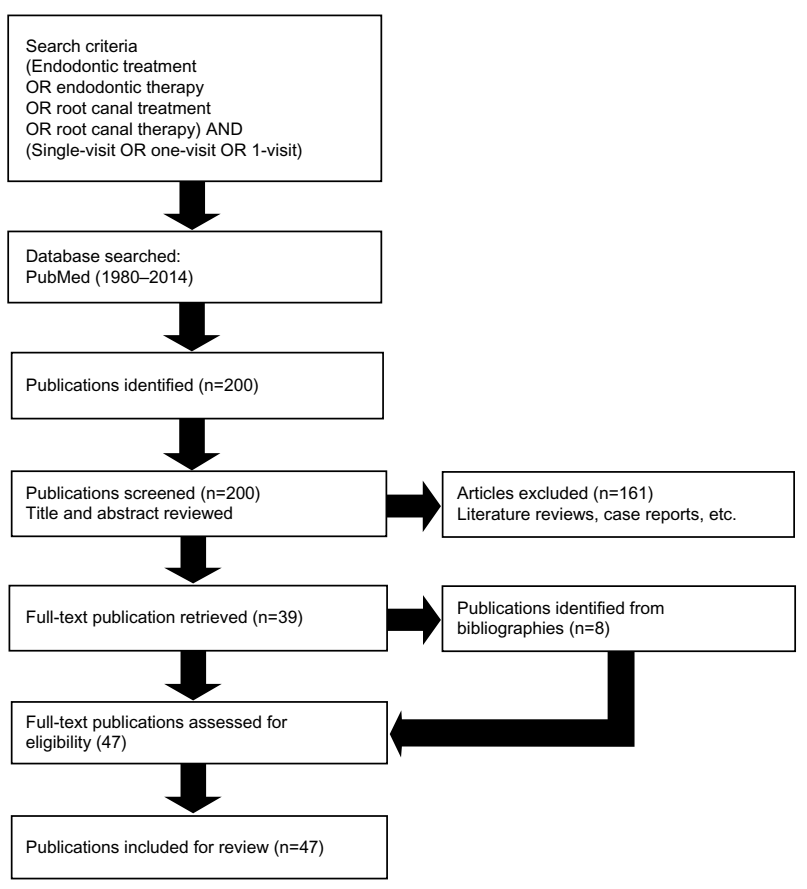

Figure I Flowchart of the literature search. tions on clinical trials. Therefore, a total of 47 publications were included in this review, and the reported postoperative complications and success rate are summarized in Tables 1 and 2 , respectively.

\section{Studies on postoperative complications of single-visit treatment}

Table 1 summarizes the studies on postoperative complications of single-visit treatments. It was reported that postoperative pain or complications were commonly found after root canal procedures, with duration ranging from one day (same day) to several weeks in the worst scenarios. The complications included swelling, tenderness to percussion, increased mobility, and systemic disturbance. The pain was reported as being from mild grade to severe forms, widely described as flare-ups. The reported findings on postoperative pain differed between studies. Many studies showed no significant differences between single-visit treatment and multiple-visit treatment. ${ }^{19-24}$ The sample sizes ranged from 32 cases to 387 cases. Among the studies, quite a number of treated teeth were limited to single-root cases.

Some studies surprisingly described more postoperative pain developing with conventional multiple-visit treatment. $^{25-28}$ One study reported slightly more postoperative pain with single-visit treatment, but that result was statistically insignificant. ${ }^{29}$ It was described ${ }^{25}$ that the incidence of pain on treated molars was double the percentage in the multiple-visit group than in the single-visit group. This does not reflect the general belief among dental practitioners. There were two studies from the literature search that reported significantly more postoperative pain for single-visit treatment. ${ }^{30,31}$ Nowadays, a large proportion of dentists seem to avoid practicing single-visit treatment, especially for molars, because they believed that doing so may lead to more postoperative pain and complications after root canal treatment.

It has been proved that postoperative pain may not correlate to age, sex, or tooth location. ${ }^{26,27}$ In another study, females were shown to have a higher incidence of postoperative pain than did male patients. ${ }^{32}$ Overinstrumentation and overfilling also showed an increase in postoperative pain. ${ }^{23}$ Postoperative pain was also reported with higher incidence in nonvital teeth in one study; ${ }^{26}$ to the contrary, another study did not show any correlation with tooth vitality status. ${ }^{27}$ The incidence of postoperative pain was reported as being higher in retreatment cases with apical periodontitis. ${ }^{27,33}$ Another study reported that a combination of calcium hydroxide and 
Table I Studies on postoperative complications with single-visit endodontic treatment

\begin{tabular}{|c|c|c|}
\hline Authors & Method & Main findings \\
\hline Akbar et $\mathrm{a}^{36}$ & $\begin{array}{l}100 \text { molars } \\
\text { Single visit }-50 \text { teeth } \\
\text { Multiple visits }-50 \text { teeth }\end{array}$ & $\begin{array}{l}\text { There was no significant difference in the flare-up rate between } \\
\text { two groups }\end{array}$ \\
\hline $\begin{array}{l}\text { Dorasani } \\
\text { et } \mathrm{al}^{37}\end{array}$ & $\begin{array}{l}64 \text { single-root teeth } \\
\text { Single visit }-34 \text { teeth } \\
\text { Multiple visits }-30 \text { teeth }\end{array}$ & $\begin{array}{l}\text { Both single-visit- and multiple-visit-treated teeth had similar clinical } \\
\text { presentation, with no significant differences }\end{array}$ \\
\hline Xavier et $\mathrm{al}^{40}$ & $\begin{array}{l}48 \text { nonvital teeth } \\
\text { Single visit }-24 \text { teeth } \\
\text { Multiple visits }-24 \text { teeth } \\
\text { Evaluation period: } 2 \text { weeks }\end{array}$ & $\begin{array}{l}\text { Both single-visit and multiple-visit endodontic treatment were effective } \\
\text { in reducing bacteria and endotoxins. Two visits were more effective } \\
\text { than one visit in reducing endotoxins }\end{array}$ \\
\hline $\begin{array}{l}\text { Bhagwat } \\
\text { and Mehta }\end{array}$ & $\begin{array}{l}60 \text { patients in single-visit treatments } \\
\text { Evaluation period: } 2 \text { weeks }\end{array}$ & $\begin{array}{l}\text { Similar incidence of pain in vital and nonvital teeth without apical } \\
\text { radiolucency. Teeth with periapical radiolucency exhibited less pain than } \\
\text { nonvital teeth without periapical radiolucency }\end{array}$ \\
\hline $\begin{array}{l}\text { Singh } \\
\text { and Garg }\end{array}$ & $\begin{array}{l}200 \text { single-root teeth } \\
\text { Single visit }-100 \text { teeth } \\
\text { Multiple visits }-100 \text { teeth } \\
\text { Evaluation period: } 2 \text { days }\end{array}$ & $\begin{array}{l}\text { No significant difference in the incidence and intensity of postobturation } \\
\text { pain experienced by the two groups }\end{array}$ \\
\hline Ali et a $\mathrm{a}^{72}$ & $\begin{array}{l}\text { I,328 patients in single-visit treatment } \\
\text { Evaluation period: } 2 \text { days }\end{array}$ & $\begin{array}{l}\text { The presence of postoperative pain was } 4 \% \\
\text { The factors that influenced the experience of postoperative pain were: } \\
\text { old age, female, mandibular teeth, and the presence of preoperative pain }\end{array}$ \\
\hline $\begin{array}{l}\text { Prashanth } \\
\text { et } \mathrm{al}^{73}\end{array}$ & $\begin{array}{l}32 \text { cases } \\
\text { Single visit }-16 \text { teeth } \\
\text { Multiple visits }-16 \text { teeth } \\
\text { Evaluation period: } 6 \text { weeks }\end{array}$ & $\begin{array}{l}\text { No significant difference in terms of success, postoperative pain, or } \\
\text { tenderness existed when treated with either single-visit or multiple-visit } \\
\text { therapy }\end{array}$ \\
\hline $\begin{array}{l}\text { Xiao and } \\
\text { Zhang }^{74}\end{array}$ & $\begin{array}{l}\text { I38 teeth } \\
\text { Single visit }-76 \text { teeth } \\
\text { Multiple visits }-62 \text { teeth } \\
\text { Evaluation period: } 2 \text { years }\end{array}$ & $\begin{array}{l}\text { Single-visit endodontic treatment had the same clinical efficacy as } \\
\text { two-visit treatment in terms of postoperative pain level and short-term } \\
\text { healing }\end{array}$ \\
\hline Wang et $\mathrm{a}^{24}$ & $\begin{array}{l}89 \text { incisors by two endodontists } \\
\text { Single visit }-43 \text { teeth } \\
\text { Multiple visits }-46 \text { teeth } \\
\text { Evaluation period: } 7 \text { days }\end{array}$ & $\begin{array}{l}\text { There was no significant difference on the incidence and severity of } \\
\text { reported postoperative pain between the two groups }\end{array}$ \\
\hline $\begin{array}{l}\text { El Mubarak } \\
\text { et } \mathrm{al}^{22}\end{array}$ & $\begin{array}{l}234 \text { teeth } \\
\text { Single visit }-32 \text { teeth } \\
\text { Multiple visits }-202 \text { teeth } \\
\text { Evaluation period: I day }\end{array}$ & $\begin{array}{l}\text { Overall incidence of postoperative pain was } 9 \% \text { after I day } \\
\text { No significant difference between the two groups }\end{array}$ \\
\hline $\begin{array}{l}\text { Kalhoro } \\
\text { and Mirza } \\
\text { Ince et } \mathrm{al}^{76}\end{array}$ & $\begin{array}{l}100 \text { patients for single-visit treatment } \\
\text { Evaluation period: I month } \\
306 \text { patients by two clinicians } \\
\text { Single visit - I } 53 \text { teeth } \\
\text { Multiple visits - I53 teeth } \\
\text { Evaluation period: } 3 \text { days }\end{array}$ & $\begin{array}{l}\text { No flare-ups in I month. It was safe in both vital and nonvital teeth, and } \\
\text { even in teeth with periapical pathosis } \\
\text { No significant difference between the two groups } \\
\text { No significant difference between vital and nonvital teeth }\end{array}$ \\
\hline Risso et $\mathrm{a}^{25}$ & $\begin{array}{l}\text { I } 18 \text { molars with necrotic pulp } \\
\text { Single visit }-57 \text { teeth } \\
\text { Multiple visits }-61 \text { teeth } \\
\text { Evaluation period: } 10 \text { days }\end{array}$ & $\begin{array}{l}\text { The frequencies of postoperative pain were } 10.5 \% \text { and } 23 \% \text { for the } \\
\text { single-visit and multiple-visit group, respectively, which were of } \\
\text { significant difference }\end{array}$ \\
\hline Lin and $\mathrm{Gao}^{77}$ & $\begin{array}{l}\text { 142 teeth } \\
\text { Single visit }-100 \text { teeth } \\
\text { Multiple visits }-42 \text { teeth } \\
\text { Evaluation period: } 6 \text { months }\end{array}$ & $\begin{array}{l}\text { No statistical significance between the two groups regarding pain after } \\
7 \text { days and healing response after } 6 \text { months }\end{array}$ \\
\hline $\mathrm{Ng}$ et $\mathrm{al}^{34}$ & $\begin{array}{l}415 \text { patients } \\
\text { Single visit }-91 \text { teeth } \\
\text { Multiple visits }-324 \text { teeth } \\
\text { Evaluation period: } 2 \text { days }\end{array}$ & $\begin{array}{l}40 \% \text { reported postoperative pain } \\
\text { Prevalence associated with the female sex, molar, size of periapical } \\
\text { lesion smaller than } 3 \mathrm{~mm} \text {, preexisting pain or swelling and single-visit } \\
\text { treatment }\end{array}$ \\
\hline $\begin{array}{l}\text { Oginni } \\
\text { and Udoye }{ }^{30}\end{array}$ & $\begin{array}{l}243 \text { teeth } \\
\text { Single visit - } 107 \text { teeth } \\
\text { Multiple visits }-136 \text { teeth } \\
\text { Evaluation period: } 30 \text { days }\end{array}$ & $\begin{array}{l}\text { Flare ups in single-visit group at a rate of } 18.3 \% \text { and in the multiple-visit } \\
\text { group at } 8.1 \% P<0.05 \text {. Higher incidences for postobturation pain were } \\
\text { observed for single-visit treatment than for multiple-visit treatment }\end{array}$ \\
\hline
\end{tabular}


Table I (Continued)

\begin{tabular}{|c|c|c|}
\hline Authors & Method & Main findings \\
\hline Yoldas et $\mathrm{al}^{3 !}$ & $\begin{array}{l}218 \text { retreatment cases } \\
\text { Single visit - } 106 \text { teeth } \\
\text { Multiple visits }-112 \text { teeth } \\
\text { Evaluation period: I week }\end{array}$ & $\begin{array}{l}\text { Multiple-visit root canal treatment was more effective in completely } \\
\text { eliminating pain than was single-visit treatment of previously } \\
\text { symptomatic teeth }\end{array}$ \\
\hline DiRenzo et $\mathrm{al}^{21}$ & $\begin{array}{l}72 \text { molars treated by two operators } \\
\text { Single visit }-39 \text { teeth } \\
\text { Multiple visits }-33 \text { teeth } \\
\text { Evaluation period: } 2 \text { days }\end{array}$ & $\begin{array}{l}\text { No difference in postoperative pain between the two groups } \\
\text { One patient (I.3\%) in the multiple-visit group with preexisting apical } \\
\text { periodontitis experienced flare-up }\end{array}$ \\
\hline $\begin{array}{l}\text { Albashaireh } \\
\text { and Alnegrish }{ }^{26}\end{array}$ & $\begin{array}{l}291 \text { teeth treated by one operator } \\
\text { Single visit - I } 42 \text { teeth } \\
\text { Multiple visits - I } 49 \text { teeth } \\
\text { Evaluation period: I month }\end{array}$ & $\begin{array}{l}\text { Multiple-visit-treated and nonvital teeth had more postoperative pain. } \\
\text { Age, sex, pulpal vitality, tooth type, and preexisting pain were not found } \\
\text { to be significant factors }\end{array}$ \\
\hline $\mathrm{Fava}^{78}$ & $\begin{array}{l}90 \text { incisors for single-visit treatment } \\
\text { Treated by one operator } \\
\text { Evaluation period: } 7 \text { days }\end{array}$ & Incidence of postoperative pain after 2 days and 7 days was $5 \%$ for both \\
\hline $\begin{array}{l}\text { Imura } \\
\text { and Zuolo27 }\end{array}$ & $\begin{array}{l}\text { I,01 } 2 \text { teeth } \\
\text { Treated by } 2 \text { dentists } \\
\text { Single visit }-582 \text { teeth } \\
\text { Multiple visits }-430 \text { teeth }\end{array}$ & $\begin{array}{l}\text { There was a significantly higher incidence of flare-ups with multiple-visit } \\
\text { than with single-visit treatment }\end{array}$ \\
\hline Fava $^{68}$ & $\begin{array}{l}60 \text { upper central incisors } \\
\text { Single visit }-30 \text { teeth } \\
\text { Multiple visits }-30 \text { teeth } \\
\text { Evaluation period: } 2 \text { days }\end{array}$ & No difference in pain incidence between the two groups \\
\hline Trope $^{33}$ & $\begin{array}{l}226 \text { teeth for single-visit treatment } \\
\text { Treated by one operator }\end{array}$ & $\begin{array}{l}\text { No flare-up in cases without preexisting symptoms } \\
\text { Higher flare-up rate in retreatment cases with preexisting symptoms }\end{array}$ \\
\hline $\mathrm{Fava}^{79}$ & $\begin{array}{l}60 \text { upper central incisors } \\
\text { Single visit }-30 \text { teeth } \\
\text { Multiple visits }-30 \text { teeth } \\
\text { Evaluation period: } 7 \text { days }\end{array}$ & $\begin{array}{l}\text { No difference was observed in the incidence of postoperative pain } \\
\text { between the two groups }\end{array}$ \\
\hline Yesilsoy et $\mathrm{al}^{80}$ & $\begin{array}{l}\text { I } 86 \text { patients } \\
\text { Single visit - } 28 \text { teeth } \\
\text { Multiple visits - I } 58 \text { teeth } \\
\text { Evaluation period: } 4 \text { days }\end{array}$ & No significant differences between the two groups \\
\hline Oliet $^{19}$ & $\begin{array}{l}387 \text { teeth treated by one operator } \\
\text { Single visit }-264 \text { teeth } \\
\text { Multiple visits - } 123 \text { teeth } \\
\text { Evaluation period: } 7 \text { days }\end{array}$ & $\begin{array}{l}\text { No significant difference on postoperative pain between the two } \\
\text { groups. More pain associated with overfilled teeth }\end{array}$ \\
\hline Roane et $\mathrm{al}^{28}$ & $\begin{array}{l}359 \text { patients } \\
\text { Single visit }-250 \text { teeth } \\
\text { Multiple visits - } 109 \text { teeth }\end{array}$ & Multiple-visit treatment had a greater incidence of postoperative pain \\
\hline Mulhern et $\mathrm{al}^{81}$ & $\begin{array}{l}60 \text { teeth treated by } 2 \text { operators } \\
\text { Single visit }-30 \text { single-root teeth } \\
\text { Multiple visits }-30 \text { single-root teeth } \\
\text { Evaluation period: } 2 \text { days }\end{array}$ & $\begin{array}{l}\text { No significant difference in the incidence of pain existed between the } \\
\text { single- and multiple-visit groups }\end{array}$ \\
\hline $\begin{array}{l}\text { Rudner } \\
\text { and Oliet }\end{array}$ & $\begin{array}{l}283 \text { cases } \\
\text { Single visit - } 98 \text { teeth } \\
\text { Multiple visits - } 185 \text { teeth }\end{array}$ & $\begin{array}{l}\text { There was no significant difference in the incidence and severity of } \\
\text { postoperative pain between the two groups }\end{array}$ \\
\hline
\end{tabular}

chlorhexidine intracanal medications was recommended to reduce postoperative pain with preexisting symptoms, in retreatment cases. ${ }^{31}$ Research should continue to attempt to discover more potent and effective antibacterial agents. Cases with preexisting symptoms were reported to lead to increased postoperative pain. . $^{27,34}$

Postoperative intolerable pain or swelling are collectively described as flare-up, which is probably one of the most concerning issues that dentists practicing single-visit treatment must deal with. ${ }^{35}$ It was reported that there was no significant difference in flare-up rates between single-visit and multi-visit root canal treatment. ${ }^{36,37}$ The prevalence of flare-ups after single-visit treatment in the published literature was none ${ }^{21}$ to minimal, at $3 \%{ }^{38}$ It was generally believed that postoperative pain was related to the residual bacterial colonies within the canals. Apart from the iatrogenic factors 
Table 2 Clinical studies on healing and success rate of single-visit endodontic treatment

\begin{tabular}{|c|c|c|}
\hline Authors & Method & Main findings \\
\hline Dorasani et al ${ }^{37}$ & $\begin{array}{l}64 \text { single root teeth } \\
\text { Single visit }-34 \text { teeth } \\
\text { Multiple visits }-30 \text { teeth }\end{array}$ & $\begin{array}{l}\text { Both single-visit and multiple-visit-treated teeth healed satisfactorily with } \\
\text { no significant differences }\end{array}$ \\
\hline Paredes-Vieyra & 282 teeth with apical periodontitis & No significant difference in healing results between the two groups \\
\hline and Enriquez ${ }^{42}$ & $\begin{array}{l}\text { Single visit }-146 \text { teeth } \\
\text { Multiple visits - I } 36 \text { teeth } \\
\text { Evaluation period: } 2 \text { years }\end{array}$ & Single-visit treatment can be as successful as multiple-visit treatment \\
\hline Penesis et $\mathrm{al}^{43}$ & $\begin{array}{l}63 \text { patients } \\
\text { Single visit }-33 \\
\text { Multiple visits }-30 \\
\text { Evaluation period: } 12 \text { months }\end{array}$ & No significant difference in success rates between the two groups \\
\hline Molander et $\mathrm{al}^{44}$ & $\begin{array}{l}\text { I0I teeth } \\
\text { Single-visit }-53 \text { teeth } \\
\text { Multiple-visit }-48 \text { teeth } \\
\text { Evaluation period: } 24 \text { months }\end{array}$ & $\begin{array}{l}\text { There was no significant difference in term of healing results between } \\
\text { single-visit and multiple-visit treatment }\end{array}$ \\
\hline Waltimo et al ${ }^{45}$ & $\begin{array}{l}50 \text { teeth with apical periodontitis } \\
\text { Single visit }-20 \text { teeth } \\
\text { Multiple visits }-30 \text { teeth } \\
\text { Evaluation period: } 52 \text { weeks }\end{array}$ & $\begin{array}{l}\text { No significant differences in periapical healing were observed between } \\
\text { the two groups }\end{array}$ \\
\hline Field et $\mathrm{al}^{49}$ & Single visit -223 teeth & $\begin{array}{l}\text { The overall success rate was } 89.2 \% \\
\text { No significant differences based on sex, age, arch, or operators } \\
\text { Anterior teeth were treated more successfully than posterior teeth }\end{array}$ \\
\hline Kvist et $\mathrm{al}^{46}$ & $\begin{array}{l}96 \text { teeth with apical periodontitis } \\
\text { Single visit }-48 \text { teeth } \\
\text { Multiple visits }-48 \text { teeth }\end{array}$ & No significant difference between the two groups \\
\hline $\begin{array}{l}\text { Peters } \\
\text { and Wesselink }{ }^{47}\end{array}$ & $\begin{array}{l}39 \text { patients treated by one operator } \\
\text { Single visit }-21 \text { teeth } \\
\text { Multiple visits }-18 \text { teeth } \\
\text { Evaluation period: } 4.5 \text { years }\end{array}$ & No significant difference in success rate between the two groups \\
\hline Trope et $\mathrm{al}^{48}$ & $\begin{array}{l}102 \text { teeth with apical periodontitis } \\
\text { Single visit }-45 \text { teeth } \\
\text { Multiple visits }-57 \text { teeth } \\
\text { Evaluation period: I year }\end{array}$ & The two groups had a similar success rate \\
\hline $\begin{array}{l}\text { Sjogren et } \mathrm{al}^{53} \\
\text { Jurcak et } \mathrm{al}^{51}\end{array}$ & $\begin{array}{l}\text { Single visit }-55 \text { single-rooted teeth } \\
102 \text { teeth } \\
\text { Single-visit treatment }\end{array}$ & $\begin{array}{l}\text { Complete periapical healing for } 5 \text { years was } 94 \% \\
\text { The overall success rate was } 89 \%\end{array}$ \\
\hline Pekruhn et al ${ }^{50}$ & $\begin{array}{l}925 \text { teeth done by one operator } \\
\text { for single-visit treatment } \\
\text { Evaluation period: I year }\end{array}$ & $\begin{array}{l}\text { The overall success rate was } 95 \% \\
\text { The incidence of failure was higher with retreatment and presence } \\
\text { of apical periodontitis }\end{array}$ \\
\hline $\begin{array}{l}\text { Southard } \\
\text { and Rooney }\end{array}$ & $\begin{array}{l}19 \text { patients single-visit treatment } \\
\text { Evaluation period: I year }\end{array}$ & $\begin{array}{l}\text { No patients experienced exacerbations of presenting signs and symptoms } \\
\text { after treatment } \\
\text { Eleven of } 19 \text { patents attended I-year recall, and they all were asymptomatic }\end{array}$ \\
\hline
\end{tabular}

generated in root canal treatment, apical periodontitis has been directly correlated with residual polymicrobial colonies and its by-products, which are embedded in the dentinal tubules. Neither single-visit treatment nor multiple-visit treatment with intracanal medications can completely eliminate microbial colonies, eg, Enterococcifaecalis..$^{39}$ It was reported that one-visit treatment may be less effective in reducing endotoxins than multiple-visit treatment. ${ }^{40}$

A meta-analysis of the selected studies was performed to compare postoperative complications of single-visit with multiple-visit endodontic treatment (Table 3). Those studies which reported only single-visit treatment were excluded in the analysis. The data were analyzed by Stata ${ }^{\circledR} 11.1$ software (StataCorp LP, College Station, TX, USA), and Figure 2 shows the results as forest plot. The results showed that the postoperative complications of single-visit and multiple-visit endodontic treatment were similar. Thus, it was concluded that there was no significant difference in postoperative complications between single-visit and multiple-visit endodontic treatment.

\section{Studies on healing and success rate of single-visit treatment}

Table 2 summarizes studies on the healing and success rate of single-visit treatment. It has been published to clarify healing 
Table 3 Meta-analysis table of clinical studies on postoperative complications of single-visit versus multiple-visit endodontic treatment

\begin{tabular}{|c|c|c|c|}
\hline Authors & $\mathbf{R R}$ & $95 \% \mathrm{Cl}$ & $\%$ weight \\
\hline Akbar et $\mathrm{al}^{36}$ & 1.250 & $0.356-4.385$ & 1.44 \\
\hline Xavier et $\mathrm{al}^{40}$ & 1.182 & $0.669-2.089$ & 4.64 \\
\hline Prashanth et $\mathrm{al}^{73}$ & 2.000 & $0.425-9.418$ & 0.99 \\
\hline Xiao and Zhang ${ }^{74}$ & 1.335 & $0.885-2.013$ & 6.41 \\
\hline Wang et $\mathrm{a}^{24}$ & 1.012 & $0.827-1.238$ & 9.33 \\
\hline El Mubarak et $\mathrm{a}^{22}$ & 0.823 & $0.262-2.584$ & 1.69 \\
\hline Ince et $\mathrm{al}^{76}$ & 1.009 & $0.87|-| .17 \mid$ & 10.01 \\
\hline Risso et $\mathrm{al}^{25}$ & 0.459 & $0.189-1.112$ & 2.55 \\
\hline Lin and $\mathrm{Gao}^{77}$ & 1.050 & $0.772-1.427$ & 7.84 \\
\hline $\mathrm{Ng}$ et $\mathrm{al}^{34}$ & 1.670 & $1.328-2.100$ & 8.96 \\
\hline Oginni and Udoye ${ }^{30}$ & 2.415 & $1.172-4.974$ & 3.43 \\
\hline Yoldas et $\mathrm{a}^{31}$ & 1.453 & $1.004-2.103$ & 6.95 \\
\hline Albashaireh and Alnegrish ${ }^{26}$ & 0.731 & $0.521-1.025$ & 7.39 \\
\hline Imura and Zuolo ${ }^{27}$ & 0.170 & $0.049-0.595$ & 1.45 \\
\hline Fava $^{68}$ & 2.000 & $0.191-20.898$ & 0.46 \\
\hline $\mathrm{Fava}^{79}$ & 3.000 & $0.127-70.829$ & 0.26 \\
\hline Yesilsoy et $\mathrm{al}^{80}$ & 0.672 & $0.291-1.549$ & 2.79 \\
\hline Oliet $^{19}$ & 1.631 & $0.766-3.473$ & 3.22 \\
\hline Roane et $\mathrm{al}^{28}$ & 0.487 & $0.325-0.730$ & 6.49 \\
\hline Mulhern et $\mathrm{al}^{81}$ & 0.667 & $0.319-1.394$ & 3.34 \\
\hline Rudner and Oliet ${ }^{20}$ & 0.974 & $0.870-1.091$ & 10.37 \\
\hline$D+L$ pooled $R R$ & 1.020 & $0.868-1.199$ & 100.00 \\
\hline
\end{tabular}

Note: $\mathrm{D}+\mathrm{L}$ refers to random effect method $^{82}$ for meta-analysis.

Abbreviations: $\mathrm{Cl}$, confidence interval; $\mathrm{RR}$, relative risk. and success rates in the context of single-visit root canal treatment. The sample size of the studies ranged from small $(n=19)$ to large $(n=925)$. They all demonstrated no significant difference in radiographic evidence of healing between singlevisit and multiple visit treatment. ${ }^{41-48} \mathrm{~A}$ study by Field et a ${ }^{49}$ reported that the success rate might be higher in the anterior teeth than the posterior teeth. They also commented that there were no significant differences in terms of the factors of sex, age, arch, and operators. Another study argued that no correlation existed between success rate and the tooth type. ${ }^{50} \mathrm{The}$ reported success rate for single-visit treatment ranged from $89 \%{ }^{51}$ to $95 \% .^{50}$ The evaluation period for the studies ranged from a minimum of 1 year ${ }^{52}$ up to 5 years. ${ }^{53}$

A meta-analysis on the selected studies was performed to compare the success rate of single-visit with multiple-visit endodontic treatment (Table 4). Those studies that reported only single-visit treatment were excluded in the analysis. The data was analyzed with Stata ${ }^{\circledR} 11.1$ software, and Figure 3 shows the results as a forest plot. The results showed that the success rates of single-visit and multiple-visit endodontic treatment were similar. Therefore, it was concluded there was no significant difference in the success rate of single-visit and multiple-visit treatment.

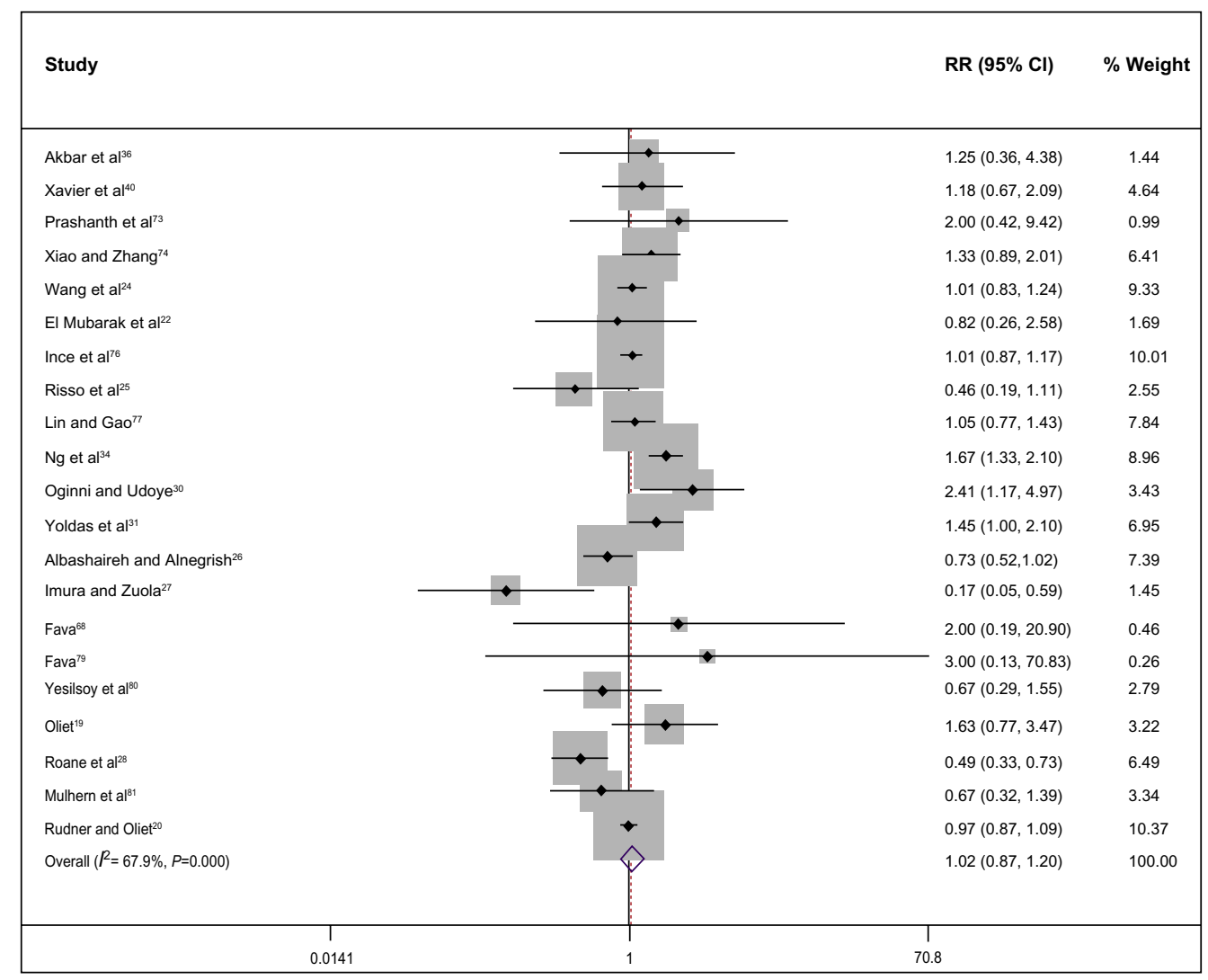

Figure 2 Forest plot for postoperative complications of single-visit versus multiple-visit endodontic treatment.

Note: Weights are from random effects analysis.

Abbreviations: $\mathrm{Cl}$, confidence interval; $\mathrm{RR}$, relative risk. 
Table 4 Meta-analysis table of clinical studies on the success rate of single-visit versus multiple-visit endodontic treatment

\begin{tabular}{llll}
\hline Authors & RR & $\mathbf{9 5 \%} \mathbf{C l}$ & \% weight \\
\hline Dorasani et al $^{37}$ & $\mathrm{I} .643$ & $0.655-4.12 \mathrm{I}$ & 15.16 \\
Paredes-Vieyra and Enriquez $^{42}$ & 0.233 & $0.026-2.058$ & 3.08 \\
Penesis et al $^{43}$ & $\mathrm{I} . \mathrm{III}$ & $0.536-2.304$ & 22.14 \\
Molander et al $^{44}$ & 1.388 & $0.717-2.685$ & 25.78 \\
Peters and Wesselink $^{47}$ & 0.686 & $0.216-2.175$ & 10.17 \\
Trope et al $^{48}$ & 0.633 & $0.315-1.273$ & 23.68 \\
D + L pooled RR & 0.992 & $0.673-1.462$ & 100.00 \\
\hline
\end{tabular}

Note: $D+L$ refers to random effect method ${ }^{82}$ for meta-analysis.

Abbreviations: $\mathrm{Cl}$, confidence interval; $\mathrm{RR}$, relative risk.

It was interesting to find that the results may contradict the general dentists' belief on root canal treatment. Single-visit treatment was generally avoided due to the possible higher failure rate for a tooth treated by single-visit procedure. The results surprisingly proved that there was no significant difference in the success rates. The preference for selection of either single-visit or multiple-visit endodontic treatment has been based on significant cultural differences. Two surveys of US endodontic teaching institutes and practicing dentists reported that $70 \%$ and $90 \%$ of respondents, respectively, would consider single-visit endodontic treatment, after selection, in certain cases. ${ }^{54,55}$ The survey found that $56 \%$ of American endodontists would complete endodontic treatment in a single visit, and 35\% would do this even on infected root canal systems. Another survey reported that a majority of the Australian endodontists would perform single-visit endodontic treatment but not as a routine practice. ${ }^{56}$ They preferred the multiple-visit approach based on their experience, unrelated to the biological concerns or patient interest. A survey in
Japan found single-visit endodontic treatment was not popular among the dentists in Japan. ${ }^{57}$

With the introduction of new technology, such as rotary nickel-titanium instruments, that can efficiently clean and shape the canals, endodontic treatment time can be substantially reduced. This allows more dentists to consider singlevisit endodontic treatment. Figini et $\mathrm{al}^{58}$ suggested that it was becoming more popular to complete the entire endodontic procedure in a single visit, in particular for endodontists and skilled general practitioners. There are a number of advantages to single-visit endodontic treatment. First, the reduced number of appointments is more convenient for patients making several visits for endodontic treatment. It reduces the need for repeated episodes of antibiotics in cardiovascularsusceptible individuals. It is also an alternative to offer for busy patients with time-restraints for treatment. Second, the reduced number of appointments allows clinicians to manage office time efficiently, by reducing time wasted on failed appointments and rescheduling. Third, single-visit endodontic treatment reduces patient discomfort and risks associated with local anesthesia. It also reduces the episodes of pain and anxiety that may arise from each appointment. Fourth, singlevisit endodontic treatment minimizes the possible chance of iatrogenic errors (eg, perforation, ledging, stripping, and extrusion of antimicrobial irrigants due to longer exposures in instrumentation procedures). Fifth, it allows dentists to obturate the canals that are well-oriented and the operator is familiar with. Sixth, with single-visit treatment, there is no need for provisional restoration between appointments and thus no bacterial contamination through the leakage beneath

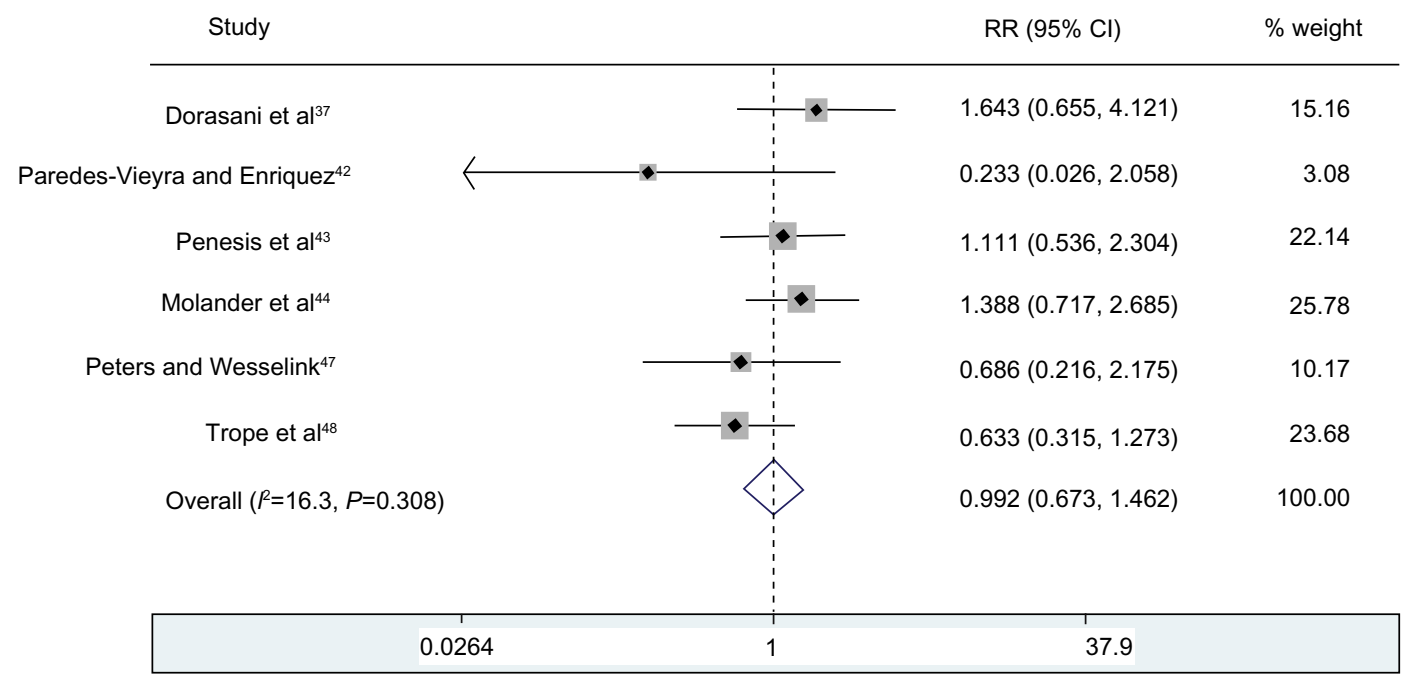

Figure 3 Forest plot for the success rate of single-visit versus multiple-visit endodontic treatment.

Note: Weights are from random effects analysis.

Abbreviations: $\mathrm{Cl}$, confidence interval; $\mathrm{RR}$, relative risk. 
the provisional restoration. Finally, single-visit endodontic treatment allows for resumption of the tooth function efficiently and immediately after treatment.

Single-visit endodontic treatment, however, has some disadvantages. Completing treatment in a single appointment may involve time restraints and causes fatigue in both the clinician and the patient. Preexisting temporomandibular joint dysfunction is a contraindication because the long treatment time can induce stress and joint dysfunction in the patient. Single-visit endodontic treatment should not be performed when the root canals cannot be dried due to exudates from the acute apical periodontitis. There are studies ${ }^{30,31,34}$ reporting an increase in postoperative pain and flare-up rate by one visit for endodontic treatment, but there are also studies ${ }^{22,24,36,80}$ reported no increase in postoperative complication.

\section{Factors to consider in performing single-visit endodontic treatment}

Ashkenaz ${ }^{15}$ suggested that the dentist's clinical experience, prescheduled appointment times, clinical techniques, restorative concerns, pulpal status, and preexisting symptoms should be considered before performing single-visit endodontic treatment. Carrotte ${ }^{59}$ proposed that it would be appropriate to proceed with obturation once the dentist has completed the root canal preparation and debridement. If the root canals cannot be dried due to persistent apical exudates, the canals should be dressed with intervisit medications, such as nonsetting calcium hydroxide. A temporary filling with a good coronal seal, such as obtained with zinc oxide and eugenol, is necessary to prevent leakage. Otherwise, there will be bacterial recolonization, which jeopardizes the success of endodontic treatment. ${ }^{60}$ Sjogren et $\mathrm{al}^{53}$ reported that the success rate in terms of periapical healing is higher ( $94 \%$ ) with a negative culture prior to obturation as compared to that with a positive culture $(68 \%)$.

Some studies reported significant disinfection of the root canals using low-energy laser photodynamic therapy. ${ }^{61-63}$ For example, Peters et $\mathrm{al}^{61}$ demonstrated that erbium: yttriumaluminum-garnet (Er:YAG) laser-pulsed irradiation considerably decreased the bacterial count in the infected root canal during endodontic treatment. Nevertheless, Meire et $\mathrm{al}^{64}$ reported that the effect of Er, chromium (Cr):yttriumscandium-gallium-garnet (YSGG) laser irradiation was less efficient than conventional sodium hypochlorite irrigation in disinfection of the contaminated root canals. Since there are limited studies in the literature, further studies are necessary to show whether laser should be used in endodontic treatment. However, it is generally agreed upon that the prepared canals can never be sterile before obturation, no matter how potent the antibacterial irrigants or intracanal medications are. If the principles of removal of microbial contaminants in the canals and dentinal walls, as much as possible by thoroughly cleaning, disinfecting, shaping, and obturation, the body's immune system would take care of the healing eventually. ${ }^{20,65}$

There are currently two measures to reduce bacterial persistence and reinfection in the canals. We can either dress the canals with antibacterial agents in multiple visits or immediately obturate the canals, to reduce the space for bacterial colonization, in a single-visit approach. Nonsetting calcium hydroxide is the most popular intracanal medication used among the variety of different antibacterial agents. ${ }^{66}$ However, its efficacy in controlling bacterial colonization has been debated. Studies have reported that the clinical outcome of multiple-visit endodontic treatment was better for teeth treated with the intracanal calcium hydroxide than for those with root canals left empty. ${ }^{48,67}$ However, other studies have suggested that the additional disinfecting effect of intracanal calcium hydroxide used in multi-visit treatment cannot be overstressed. ${ }^{45,68}$ Despite the high alkaline antibacterial properties of calcium hydroxide, some bacteria species, such as E. faecalis and Candida albicans, have been found to be resistant to it. ${ }^{45}$ Chong and Pitt Ford ${ }^{69}$ questioned the efficacy of nonsetting calcium hydroxide as a dressing in endodontic treatment. It is therefore generally considered that nonsetting calcium hydroxide should be used as a supplement to antibacterial irrigations.

Single-visit endodontic treatment and multiple-endodontic treatment have their advantages and disadvantages. In general, many dentists considered the single-visit approach to be an alternative to a multiple-visit but have no trouble replacing it. The success of endodontic treatment should be based on careful case selection. There should be no shortcuts in any of the steps throughout the treatment procedures. Clinicians should evaluate their own clinical skills and the needs of the patient. Notwithstanding the single-visit treatment approach, the clinicians should directly follow endodontic principles.

\section{Conclusion}

In conclusion, the studies reported in the literature showed that neither single-visit endodontic treatment nor multiple-visit treatment could be carried out with consequent induction of postoperative pain. Similarly neither single-visit endodontic treatment nor multiple-visit treatment has superior results over the other in terms of healing or success rate. The sample size of many studies was small, and studies with a large enough sample size for statistical analysis should be 
performed. In addition, a prospective, randomized clinical trial is needed to further verify the postoperative pain and success rate of single-visit versus multiple-visit endodontic treatment.

\section{Acknowledgments}

The authors wish to thank Miss Samantha KY Li for her assistance with the statistics used in this review.

\section{Disclosure}

The authors report no conflicts of interest in this work.

\section{References}

1. Mohammadi Z, Farhad A, Tabrizizadeh M. One-visit versus multiple-visit endodontic therapy - a review. Int Dent J. 2006;56(5): 289-293.

2. Goldfein J, Speirs C, Finkelman M, Amato R. Rubber dam use during post placement influences the success of root canal-treated teeth. J Endod. 2013;39(12):1481-1484.

3. AAE Special Committee to Develop a Microscope Position Paper. AAE Position Statement. Use of microscopes and other magnification techniques. J Endod. 2012;38(8):1153-1155.

4. Del Fabbro M, Taschieri S, Lodi G, Banfi G, Weinstein RL. Magnification devices for endodontic therapy [review]. Cochrane Database Syst Rev. 2009;8:CD005969.

5. Parekh V, Taluja C. Comparative study of periapical radiographic techniques with apex locator for endodontic working length estimation: an ex vivo study. J Contemp Dent Pract. 2011;12(2):131-134.

6. Cheung GS, Liu CS. A retrospective study of endodontic treatment outcome between nickel-titanium rotary and stainless steel hand filing techniques. J Endod. 2009;35(7):938-943.

7. Dodge JS. Immediate root filling. Dental Cosmos. 1887;29:234-235.

8. Kells CE. Immediate root filling. Dental Cosmos. 1887;29:366-367.

9. Turner WJ. New York Odontological Society. Dent Cosmos. 1901;43: $1405-1412$.

10. Hofheinz RH. Immediate root filling. Dental Cosmos. 1892;34: $182-187$.

11. Ferranti P. Treatment of the root canal of an infected tooth in one appointment: a report of 340 cases. Dent Dig. 1959;65:490-494.

12. Tosti A. Immediate endodontics: one-visit technic. Dent Surv. 1970;46(4):24-26.

13. Gutmann JL. Clinical, radiographic, and histologic perspectives on success and failure in endodontics. Dent Clin North Am. 1992;36(2): 379-392.

14. Grossman LI. Rationale of endodontic treatment. Dent Clin North Am. 1967:483-490.

15. Ashkenaz PJ. One-visit endodontics. Dent Clin North Am. 1984; 28(4):853-863.

16. Grossman LI. Endodontics 1776-1976: a bicentennial history against the background of general dentistry. J Am Dent Assoc. 1976;93(1): 78-87.

17. Morgan LF, Montgomery S. An evaluation of the crown-down pressureless technique. J Endod. 1984;10(10):491-498.

18. Carrotte P. 21st century endodontics: part 3. Int Dent J. 2005;55(4): 247-253.

19. Oliet S. Single-visit endodontics: a clinical study. J Endod. 1983;9(4):147-152.

20. Rudner WL, Oliet S. Single-visit endodontics: a concept and a clinical study. Compend Contin Educ Dent. 1981;2(2):63-68.

21. DiRenzo A, Gresla T, Johnson BR, Rogers M, Tucker D, BeGole EA. Postoperative pain after 1- and 2-visit root canal therapy. Oral Surg Oral Med Oral Pathol Oral Radiol Endod. 2002;93(5):605-610.
22. El Mubarak AH, Abu-bakr NH, Ibrahim YE. Postoperative pain in multiple-visit and single-visit root canal treatment. $J$ Endod. 2010;36(1):36-39.

23. Gesi A, Hakeberg M, Warfvinge J, Bergenholtz G. Incidence of periapical lesions and clinical symptoms after pulpectomy - a clinical and radiographic evaluation of 1- versus 2-session treatment. Oral Surg Oral Med Oral Pathol Oral Radiol Endod. 2006;101(3):379-388.

24. Wang C, Xu P, Ren L, Dong G, Ye L. Comparison of post-obturation pain experience following one-visit and two-visit root canal treatment on teeth with vital pulps: a randomized controlled trial. Int Endod J. 2010;43(8):692-697.

25. Risso PA, Cunha AJ, Araujo MC, Luiz RR. Postobturation pain and associated factors in adolescent patients undergoing one- and two-visit root canal treatment. J Dent. 2008;36(11):928-934.

26. Albashaireh ZS, Alnegrish AS. Postobturation pain after single- and multiple-visit endodontic therapy. A prospective study. J Dent. 1998;26(3):227-232.

27. Imura N, Zuolo ML. Factors associated with endodontic flare-ups: a prospective study. Int Endod J. 1995;28(5):261-265.

28. Roane JB, Dryden JA, Grimes EW. Incidence of postoperative pain after single- and multiple-visit endodontic procedures. Oral Surg Oral Med Oral Pathol. 1983;55(1):68-72.

29. Pekruhn RB. Single-visit endodontic therapy: a preliminary clinical study. J Am Dent Assoc. 1981;103(6):875-877.

30. Oginni A, Udoye CI. Endodontic flare-ups: comparison of incidence between single and multiple visits procedures in patients attending a Nigerian teaching hospital. Odontostomatol Trop. 2004;27(108):23-27.

31. Yoldas O, Topuz A, Isçi AS, Oztunc H. Postoperative pain after endodontic retreatment: single- versus two-visit treatment. Oral Surg Oral Med Oral Pathol Oral Radiol Endod. 2004;98(4):483-487.

32. Fox J, Atkinson JS, Dinin AP, et al. Incidence of pain following one-visit endodontic treatment. Oral Surg Oral Med Oral Pathol. 1970;30(1):123-130.

33. Trope M. Flare-up rate of single-visit endodontics. Int Endod J. 1991;24(1):24-26.

34. Ng YL, Glennon JP, Setchell DJ, Gulabivala K. Prevalence of and factors affecting post-obturation pain in patients undergoing root canal treatment. Int Endod J. 2004;37(6):381-391.

35. Buchanan LS. One-visit endodontics: a new model of reality. Dent Today. 1996;15(5):36, 38, 40-36, 38, 43.

36. Akbar I, Iqbal A, Al-Omiri MK. Flare-up rate in molars with periapical radiolucency in one-visit vs two-visit endodontic treatment. J Contemp Dent Pract. 2013;14(3):414-418.

37. Dorasani G, Madhusudhana K, Chinni SK. Clinical and radiographic evaluation of single-visit and multi-visit endodontic treatment of teeth with periapical pathology: An in vivo study. J Conserv Dent. 2013;16(6):484-488.

38. Eleazer PD, Eleazer KR. Flare-up rate in pulpally necrotic molars in one-visit versus two-visit endodontic treatment. J Endod. 1998;24(9): 614-616.

39. Gurgel-Filho ED, Vivacqua-Gomes N, Gomes BP, Ferraz CC, Zaia AA, Souza-Filho FJ. In vitro evaluation of the effectiveness of the chemomechanical preparation against Enterococcus faecalis after single- or multiple-visit root canal treatment. Braz Oral Res. 2007;21(4): 308-313.

40. Xavier AC, Martinho FC, Chung A, et al. One-visit versus two-visit root canal treatment: effectiveness in the removal of endotoxins and cultivable bacteria. $J$ Endod. 2013;39(8):959-964.

41. K B. Trial suggests no difference between single-visit and two-visit root canal treatment. Evid Based Dent. 2013;14(2):48.

42. Paredes-Vieyra J, Enriquez FJ. Success rate of single- versus two-visit root canal treatment of teeth with apical periodontitis: a randomized controlled trial. $J$ Endod. 2012;38(9):1164-1169.

43. Penesis VA, Fitzgerald PI, Fayad MI, Wenckus CS, BeGole EA, Johnson BR. Outcome of one-visit and two-visit endodontic treatment of necrotic teeth with apical periodontitis: a randomized controlled trial with oneyear evaluation. $J$ Endod. 2008;34(3):251-257. 
44. Molander A, Warfvinge J, Reit C, Kvist T. Clinical and radiographic evaluation of one- and two-visit endodontic treatment of asymptomatic necrotic teeth with apical periodontitis: a randomized clinical trial. J Endod. 2007;33(10):1145-1148.

45. Waltimo T, Trope M, Haapasalo M, Ørstavik D. Clinical efficacy of treatment procedures in endodontic infection control and one year follow-up of periapical healing. J Endod. 2005;31(12):863-866.

46. Kvist T, Molander A, Dahlén G, Reit C. Microbiological evaluation of one- and two-visit endodontic treatment of teeth with apical periodontitis: a randomized, clinical trial. J Endod. 2004;30(8): 572-576.

47. Peters LB, Wesselink PR. Periapical healing of endodontically treated teeth in one and two visits obturated in the presence or absence of detectable microorganisms. Int Endod J. 2002;35(8):660-667.

48. Trope M, Delano EO, Orstavik D. Endodontic treatment of teeth with apical periodontitis: single vs multivisit treatment. J Endod. 1999;25(5):345-350.

49. Field JW, Gutmann JL, Solomon ES, Rakusin H. A clinical radiographic retrospective assessment of the success rate of single-visit root canal treatment. Int Endod J. 2004;37(1):70-82.

50. Pekruhn RB. The incidence of failure following single-visit endodontic therapy. J Endod. 1986;12(2):68-72.

51. Jurcak JJ, Bellizzi R, Loushine RJ. Successful single-visit endodontics during Operation Desert Shield. J Endod. 1993;19(8):412-413.

52. Southard DW, Rooney TP. Effective one-visit therapy for the acute periapical abscess. J Endod. 1984;10(12):580-583.

53. Sjögren U, Figdor D, Persson S, Sundqvist G. Influence of infection at the time of root filling on the outcome of endodontic treatment of teeth with apical periodontitis. Int Endod J. 1997;30(5):297-306.

54. Calhoun RL, Landers RR. One-appointment endodontic therapy: a nationwide survey of endodontists. J Endod. 1982;8(1):35-40.

55. Landers RR, Calhoun RL. One-appointment endodontic therapy: an opinion survey. J Endod. 1980;6(10):799-801.

56. Sathorn C, Parashos P, Messer H. Australian endodontists' perceptions of single and multiple visit root canal treatment. Int Endod J. 2009;42(9):811-818

57. Inamoto K, Kojima K, Nagamatsu K, Hamaguchi A, Nakata K, Nakamura H. A survey of the incidence of single-visit endodontics. J Endod. 2002;28(5):371-374.

58. Figini L, Lodi G, Gorni F, Gagliani M. Single versus multiple visits for endodontic treatment of permanent teeth: a Cochrane systematic review. J Endod. 2008;34(9):1041-1047.

59. Carrotte P. 21st century endodontics. Part 4. Int Dent J. 2005;55(5): 334-340.

60. Saunders WP, Saunders EM. Coronal leakage as a cause of failure in root-canal therapy: a review. Endod Dent Traumatol. 1994;10(3): $105-108$

61. Peters OA, Bardsley S, Fong J, Pandher G, Divito E. Disinfection of root canals with photon-initiated photoacoustic streaming. J Endod. 2011;37(7):1008-1012.

62. Ng R, Singh F, Papamanou DA, et al. Endodontic photodynamic therapy ex vivo. J Endod. 2011;37(2):217-222.

63. Garcez AS, Nuñez SC, Hamblim MR, Suzuki H, Ribeiro MS. Photodynamic therapy associated with conventional endodontic treatment in patients with antibiotic-resistant microflora: a preliminary report. J Endod. 2010;36(9):1463-1466.

64. Meire MA, De Prijck K, Coenye T, Nelis HJ, De Moor RJ. Effectiveness of different laser systems to kill Enterococcus faecalis in aqueous suspension and in an infected tooth model. Int Endod J. 2009;42(4): 351-359.
65. Kenrick S. Endodontics: a multiple-visit or single-visit approach. Aust Endod J. 2000;26(2):82-85.

66. Kawashima N, Wadachi R, Suda H, Yeng T, Parashos P. Root canal medicaments. Int Dent J. 2009;59(1):5-11.

67. Ghoddusi J, Javidi M, Zarrabi MH, Bagheri H. Flare-ups incidence and severity after using calcium hydroxide as intracanal dressing. $N Y$ State Dent J. 2006;72(4):24-28.

68. Fava LR. A clinical evaluation of one and two-appointment root canal therapy using calcium hydroxide. Int Endod J. 1994;27(1):47-51.

69. Chong BS, Pitt Ford TR. The role of intracanal medication in root canal treatment. Int Endod J. 1992;25(2):97-106.

70. Bhagwat $S$, Mehta $D$. Incidence of post-operative pain following single visit endodontics in vital and non-vital teeth: An in vivo study. Contemp Clin Dent. 2013;4(3):295-302.

71. Singh S, Garg A. Incidence of post-operative pain after single visit and multiple visit root canal treatment: A randomized controlled trial. J Conserv Dent. 2012;15(4):323-327.

72. Ali SG, Mulay S, Palekar A, Sejpal D, Joshi A, Gufran H. Prevalence of and factors affecting post-obturation pain following single visit root canal treatment in Indian population: A prospective, randomized clinical trial. Contemp Clin Dent. 2012;3(4):459-463.

73. Prashanth MB, Tavane PN, Abraham S, Chacko L. Comparative evaluation of pain, tenderness and swelling followed by radiographic evaluation of periapical changes at various intervals of time following single and multiple visit endodontic therapy: an in vivo study. J Contemp Dent Pract. 2011;12(3):187-191.

74. Xiao D, Zhang DH. [A clinical study of one-visit endodontic treatment for infected root canals]. Hua Xi Kou Qiang Yi Xue Za Zhi. 2010;28(1): 57-60. Chinese.

75. Kalhoro FA, Mirza AJ. A study of flare-ups following single-visit root canal treatment in endodontic patients. J Coll Physicians Surg Pak. 2009;19(7):410-412.

76. Ince B, Ercan E, Dalli M, Dulgergil CT, Zorba YO, Colak H. Incidence of postoperative pain after single- and multi-visit endodontic treatment in teeth with vital and non-vital pulp. Eur J Dent. 2009;3(4):273-279.

77. Lin NY, Gao XJ. [A short-term clinical study of one-visit endodontic treatment for infected root canals]. Zhonghua Kou Qiang Yi Xue Za Zhi. 2006;41(9):525-528. Chinese.

78. Fava LR. Single visit root canal treatment: incidence of postoperative pain using three different instrumentation techniques. Int Endod J. 1995;28(2):103-107.

79. Fava LR. A comparison of one versus two appointment endodontic therapy in teeth with non-vital pulps. Int Endod J. 1989;22(4):179-183.

80. Yesilsoy C, Koren LZ, Morse DR, Rankow H, Bolanos OR, Furst ML. Post-endodontic obturation pain: a comparative evaluation. Quintessence Int. 1988;19(6):431-438.

81. Mulhern JM, Patterson SS, Newton CW, Ringel AM. Incidence of postoperative pain after one-appointment endodontic treatment of asymptomatic pulpal necross in single-rooted teeth. J Endod. 1982;8(8):370-375.

82. DerSimonian R, Laird N. Meta-analysis in clinical trials. Controlled clinical trials. 1986;7(3):177-188. 
Clinical, Cosmetic and Investigational Dentistry

Dovepress

\section{Publish your work in this journal}

Clinical, Cosmetic and Investigational Dentistry is an international, peer-reviewed, open access, online journal focusing on the latest clinical and experimental research in dentistry with specific emphasis on cosmetic interventions. Innovative developments in dental materials, techniques and devices that improve outcomes and patient satisfaction

and preference will be highlighted. The manuscript management system is completely online and includes a very quick and fair peerreview system, which is all easy to use. Visit http://www.dovepress. com/testimonials.php to read real quotes from published authors.

Submit your manuscript here: http://www.dovepress.com/clinical-cosmetic-and-investigational-dentistry-journal 